\title{
The Role of Grain Boundary Dislocations in the Segregation-Induced Grain Boundary Embrittlement of Copper by Bismuth
}

\author{
C. A. Wade ${ }^{1,2}$, I. MacLaren ${ }^{3}$, R.P. Vinci ${ }^{1}$, and M. Watanabe ${ }^{1}$ \\ ${ }^{1}$ Materials Science and Engineering Department, Lehigh University, Bethlehem, PA, USA \\ ${ }^{2}$ School of Materials, University of Manchester, Manchester M1 3BB, UK \\ ${ }^{3}$ School of Physics and Astronomy, University of Glasgow, Glasgow G12 8QQ, UK
}

The mechanical embrittlement of copper $(\mathrm{Cu})$ by bismuth $(\mathrm{Bi})$ has long been known to be a direct result of $\mathrm{Bi}$ segregation to the $\mathrm{Cu}$ grain boundaries (GBs) [1]. Correlated micro-mechanical testing and micro/nano-characterization via electron microscopy, both made possible by focused ion beam (FIB) specimen generation, has allowed the study of specific $\mathrm{Cu}$ GBs so that the mechanical behaviour of individual GBs may be linked to micro and nano-characteristics of the GBs. One such observation resulting from these correlated micro-mechanical testing and micro/nano-characterization studies has been the appearance of $\mathrm{GB}$ dislocations (GBDs) along some $\mathrm{Cu}$ GBs corresponding to reduced fracture toughness of the $\mathrm{Cu}$ GB in the presence of $\mathrm{Bi}$ [2]. At the GBs new arrangements of GBDs can be generated (defined as secondary GBSs) to accommodate the crystal lattice strain resulting from a modest departure in crystal misorientations between the two crystals comprising the GB from those orientations that provide good coincident site lattice agreement and a corresponding low GB energy [3].

In this study the $\mathrm{GB}$ structures of a $\mathrm{Bi}$ doped $38^{\circ}[1,0,0]$ twist $\mathrm{Cu}$ bicrystal have been studied to observe the interaction between the Bi segregant atoms and the secondary GBDs contained in the Cu bicrystal GB. Figure 1 shows a high-angle annular dark-field (HAADF) scanning transmission electron microscopy (STEM) image of a Bi doped $38^{\circ}[1,0,0]$ twist $\mathrm{Cu}$ bicrystal GB with a region of interest (ROI) expanded and shown as HAADF-STEM, fast Fourier transformation (FFT) filtered, and annular bright-field STEM images with the location of $\mathrm{Bi}$ as well as the additional half plane of atoms associated with an edge dislocation marked on both the filtered and raw HAADF images. In this figure it can be seen that the large $\mathrm{Bi}$ atoms that have segregated to the GB exist near the dislocation cores of the edge character dislocations inhabiting the GB. Though the GB used in this study is nominally a pure twist bicrystal, detailed convergent beam electron diffraction (CBED) analysis can be used to accurately measure both the angle of misorientation between the two crystals that make up the GB and the axis about which that rotation is applied.

The result of this misorientation analysis finds that the nominally $38^{\circ}[1,0,0]$ pure twist GB actually contains a small amount of tilt character of about $4.7^{\circ}$ about $[0,-0.44,-0.89]$. This tilt misorientation between the two grains forming the bicrystal must be accommodated by the introduction of a periodic array of edge dislocations along the GB. If this is decomposed into edge dislocations with a Burgers vector of $1 / 2<110>$ lying along two orthogonal $<001>$ directions, then one would lie along [010] with a spacing of $7.1 \mathrm{~nm}$, and the other along [001] with a spacing of $3.5 \mathrm{~nm}$. It is these edge dislocations that have been imaged via HAADF and BF-STEM and can be seen to have Bi atoms along or near the edge dislocation cores. The measured spacing of these edge dislocations along the GB via TEM and HAADFSTEM of 3 to $4 \mathrm{~nm}$ is in good agreement with the calculated spacing of these edge dislocations from CBED misorientation measurements of $3.5 \mathrm{~nm}$. The Bi interaction with the edge dislocation cores along with the edge dislocation spacing as a function of GB tilt character is useful in interpreting and understanding the observed mechanical behaviour of the GB in the corollary micro-mechanical testing 
specimens. The degree to which a $\mathrm{Cu}$ GB of primarily twist character may become embrittled is related to the availability of $\mathrm{Bi}$ to enter the GB and occupy some key sites in the cores of edge dislocations on the GB. These edge dislocation cores are then locked by the Bi preventing the emission of crack tip blunting dislocations when a tensile stress is applied normal to the GB plane. Any secondary GBDs in the twist GBs that are primarily screw in character would still be free to relieve stress at the GB through dislocation motion as these dislocations do not possess the free volume at their cores to accommodate Bi atoms. The different interactions between $\mathrm{Bi}$ and these two types of secondary GBDs present (due to the specific misorientation of a given GB) determine the degree to which a $\mathrm{Cu}$ GB may become embrittled by $\mathrm{Bi}$. The pinning of the edge dislocations and not the screw dislocations along the $38^{\circ}[1,0,0]$ explains the reduction in fracture toughness (a sign of GB embrittlement due to the restraint of the edge dislocations) along with the still prominent plastic elongation (due to the inability to pin the majority screw dislocations) observed in the micro-mechanical testing of the $\mathrm{Bi}$ doped $38^{\circ}[1,0,0] \mathrm{Cu}$ bicrystals compared to pure $\mathrm{Cu}$ bicrystals.

\section{References:}

[1] B.D. Powell and H. Mykura, Acta Met 21 (1973), p. 1151-1156.

[2] M. McLean, C.A. Wade, R.P. Vinci and M. Watanabe, Exp Mech 54 (2014), p. 685-688.

[3] T. Schober and R.W. Balluffi, Phil Mag 24 (1971), p. 165-180.
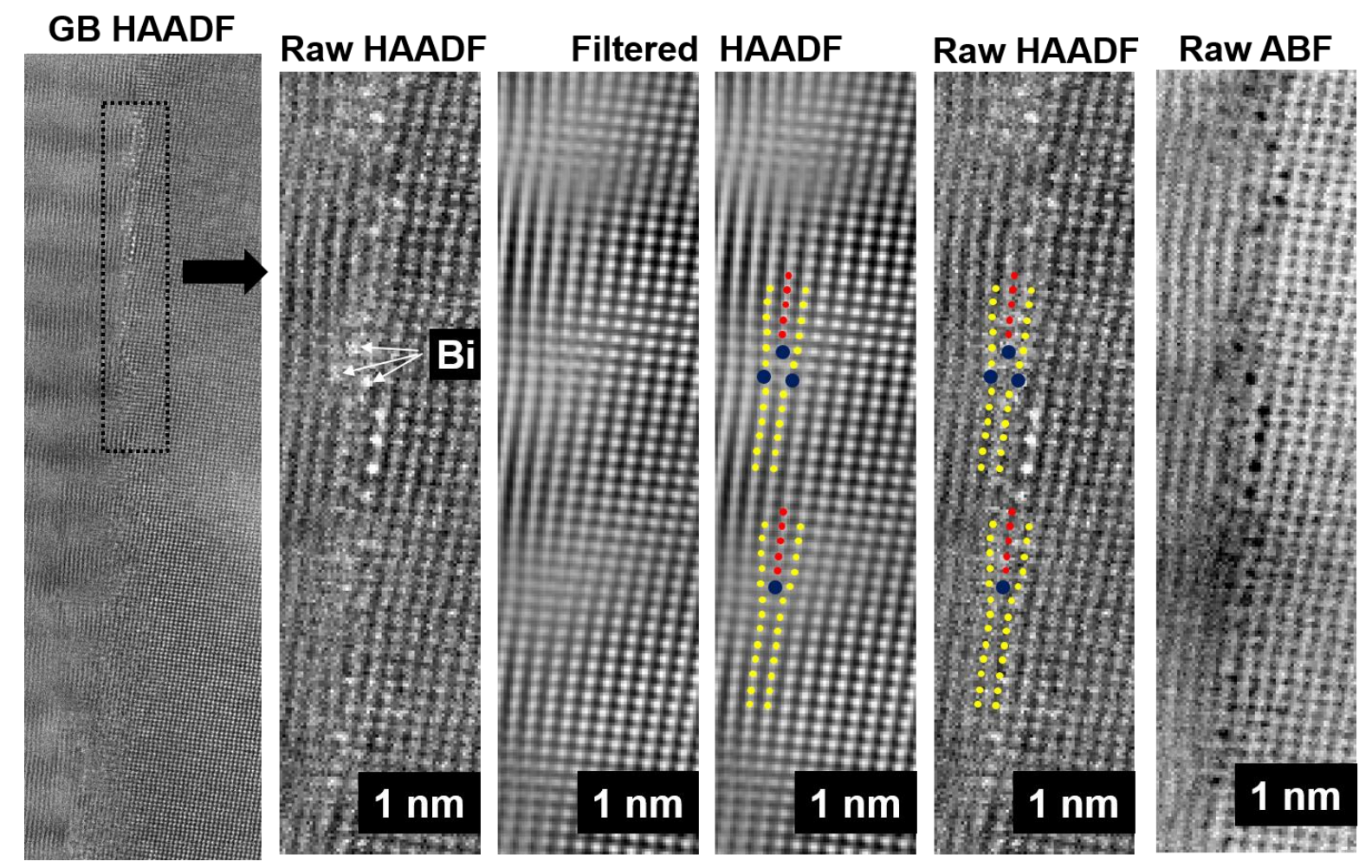

Figure 1. HAADF-STEM image of a Bi doped $38^{\circ}[1,0,0] \mathrm{Cu}$ bicrystal GB with an enlarged ROI displaying $\mathrm{Bi}$ atoms along the GB. The FFT filtered images help show the location of edge dislocation cores with the extra half plane of atoms displayed in red along the $\mathrm{Bi}$ atoms around the dislocation cores shown in blue on both the FFT filtered images and the HAADF-STEM image. An ABF-STEM image of the ROI is also provided for comparison. 